\title{
Hidden diversity of myxomycetes: problems and perspectives
}

\author{
O.N. Shchepin ${ }^{1,3 *}$, Y.K. Novozhilov ${ }^{1}$, I.S. Prikhodko ${ }^{2}$, M. Schnittler ${ }^{3}$ \\ ${ }^{1}$ Komarov Botanical Institute of the Russian Academy of Sciences, Saint Petersburg, Russia \\ ${ }^{2}$ St. Petersburg State University, Saint Petersburg, Russia \\ ${ }^{3}$ Institute of Botany and Landscape Ecology, EMA University of Greifswald, Greifswald, Germany \\ *e-mail:ledum_laconicum@mail.ru
}

Key words: myxomycetes, hidden diversity, species concepts

Motivation and Aim: Myxomycetes (= Myxogastria, plasmodial slime molds) represent a monophyletic group of free-living amoeboid protists within the supergroup Amoebozoa that are characterized by a unique life cycle with an alternation of uninucleate myxamoebae/swarm cells, multinucleate plasmodia and fruiting bodies (sporocarps) filled with airborne spores. Since Linnean times about 1000 species were validly described within five orders based almost exclusively on morphological characters of the sporocarps. However, DNA sequence-based studies revealed a paraphyly of many taxonomical groups within the class as well as an unexpected extent of hidden diversity. The direction of further research in the field of myxomycete taxonomy and diversity studies using modern approaches should be outlined.

Results and discussion: In recent years, a number of molecular phylogenetic studies revealed two problems of the morphological approach. 1. The long established five-order system does not reflect correctly the phylogenetic relationships within the class. This could be solved by creating a system based on the analysis of multiple gene markers, e. $g$. transcriptomic or genomic data. At the moment, transcriptomic data are available for only four myxomycetes species and genomic data for one. 2. Many morphospecies seem to comprise several, reproductively isolated, biospecies; others are even paraphyletic [1]. Application of DNA barcoding [2] and 18S amplicon metagenomics [3] provided evidence that diversity assessments based on morphological determination of sporocarps underestimate the diversity of the group. The presence of phylogenetic clades consisting of OTUs not matching to any known species, as well as several reports of myxamoebal strains with unknown species identity isolated from unusual habitats lend evidence for a significant, yet undescribed diversity of myxomycetes which presumably never or rarely form sporocarps. However, the incompleteness of the currently existing database of reference sequences does not allow us to distinguish such species reliably.

Acknowledgements: Supported by the RFBR (15-29-02622, 18-04-01232 A), by Presidium of UB RAS (12-M-14-2009, 12-П-4-1067) and by German DFG (SCHN 1080/2, RTG 2010).

\section{References}

1. Schnittler M., Shchepin O.N., Dagamac N.H., Borg Dahl M. et al. (2017) Barcoding myxomycetes with molecular markers: challenges and opportunities. Nova Hedwigia. 104(1-3):323-341.

2. Shchepin O.N., Dagamac N.H., Sanchez O.M., Novozhilov Y.K. et al. (2017) DNA barcoding as a tool for identification of plasmodia and sclerotia of myxomycetes (Myxogastria) appearing in moist chamber cultures. Mycosphere. 8(10):1904-1913.

3. Borg Dahl M., Brejnrod A.D., Unterseher M., Hoppe T. et al. (2017) Genetic barcoding of dark-spored myxomycetes (Amoebozoa) - identification, evaluation and application of a sequence similarity threshold for species differentiation in NGS studies. Molecular Ecology Resources. 18(2):306-318. 